\title{
The effect of memory and active forces on transition path times distributions
}

\author{
E. Carlon, ${ }^{*, \dagger}$ H. Orland, ${ }^{*,+}, \boldsymbol{T}$ T. Sakaue, ${ }^{*, \S, \|}$ and C. Vanderzande ${ }^{*, \perp, \dagger}$ \\ $\dagger K U$ Leuven, Institute for Theoretical Physics, Celestijnenlaan 200D, 3001 Leuven, \\ Belgium \\ $\ddagger$ Institut de Physique Théorique, CEA, CNRS, UMR3681, F-91191 Gif-sur-Yvette, France \\ ฯ Beijing Computational Science Research Center, No.10 East Xibeiwang Road, Beijing \\ 100193, China \\ $\S$ Department of Physics and Mathematics, Aoyama Gakuin University, 5-10-1 Fuchinobe, \\ Chuo-ku, Sagamihara, Kanagawa 252-5258, Japan \\ \|PRESTO, Japan Science and Technology Agency (JST), 4-1-8 Honcho Kawaguchi, \\ Saitama 332-0012, Japan \\ $\perp$ Faculty of Sciences, Hasselt University, 3590 Diepenbeek, Belgium \\ E-mail: enrico.carlon@kuleuven.be; henri.orland@cea.fr; sakaue@phys.aoyama.ac.jp; \\ carlo.vanderzande@uhasselt.be
}

\begin{abstract}
An analytical expression is derived for the transition path time distribution for a onedimensional particle crossing of a parabolic barrier. Two cases are analyzed: (i) A nonMarkovian process described by a generalized Langevin equation with a power-law memory kernel and (ii) a Markovian process with a noise violating the fluctuation-dissipation theorem, modeling the stochastic dynamics generated by active forces. In the case (i) we show that the anomalous dynamics strongly affecting the short time behavior of the distributions, but this happens only for very rare events not influencing the overall statistics. At long times the decay is always exponential, in disagreement with a recent study suggesting a stretched exponential decay. In the case (ii) the active forces do not substantially modify the short time behavior of the distribution, but lead to an overall decrease of the average transition path time. These findings offer some novel insights, useful for the analysis of experiments of transition path times in (bio)molecular systems.
\end{abstract}

\section{Introduction}

Biomolecular folding involves structural transitions of various time- and lengthscales. A simplified description of this process employs a single reaction coordinate performing stochastic dynamics along a free energy landscape. In the case of a two state folding, the folded and unfolded states correspond to two free energy minima, which are separated by a barrier. Typically, this barrier is high compared to the characteristic thermal energy $k_{B} T$, therefore the molecule spends the predominant fraction of its time close to one of the minima. 1 Transition paths are the part of the stochastic trajectory corresponding to an actual barrier crossing event. ${ }^{2}$ Although the transition paths correspond to a tiny fraction of the stochastic trajectory, they encompass all the information of the folding process. Measuring their duration has been for long time a big challenge, owing to the high time resolution needed. In the past few years, however, experiments have sufficiently progressed to make measurements of transition path times in nucleic acids and protein fold- 
ing possible. ${ }^{3} \sqrt[6]{6}$ Recently also the full probability distribution function of transition path times, obtained from the statistics of a large number of events, was determined.7 The theory of transition path times have been discussed in several papers. ${ }^{8} 18$ These studies mostly employed memoryless Markovian dynamics, while correlated noise, leading to memory effects and anomalous dynamics, was only considered in a few recent works. $19 \mid 20$

Anomalous dynamics is ubiquitous in macromolecular systems as polymers, as it is known from many examples. 21 This dynamics is characterized by a mean-square displacement of a suitable reaction coordinate scaling as $\left\langle\Delta x^{2}\right\rangle \sim t^{\alpha}$, with $\alpha \neq 1$. The analysis of the effect of an underling anomalous dynamics on transition path times is therefore an interesting case to study, which is one of the aims of this paper. Another purpose of the present work is to analyze transition path times for stochastic processes in which the noise has a non-thermal component, ie not satisfing a fluctuation-dissipation relation. Such noise has been used in the description of the dynamics of active systems. $\frac{28}{28}$ Our primary interest is to calculate the transition path time (TPT) distribution for these two cases and discuss the differences with the more standard situation of Markovian dynamics in thermal systems. We consider here a parabolic barrier, which leads to a dynamics described by linear stochastic differential equations and to Gaussian processes. We show that, using the formalism developed recently in Ref., $\frac{18}{18}$ the calculations are manageable and lead to some simple expressions for the TPT distributions. We discuss here several features of these distributions such as the short and long time behavior in the limit of high barriers.

\section{Generalities}

We consider a particle performing a stochastic dynamics on an inverted parabolic potential barrier $V(x)=-k x^{2} / 2$, with $k>0$. At time $t=0$ the particle starts from a point $-x_{0}+\varepsilon$. Transition paths are those paths reaching $x_{0}$ at the right side of the barrier without recrossing $-x_{0}$ and $x_{0}$. To compute the distribution of their duration one should solve the Langevin equation imposing absorbing boundary conditions in $-x_{0}$ and $x_{0}$. Free boundary conditions are however easier to handle and provide a good approximation if the barrier is high, $\frac{10}{10}$ i.e. $\beta E \gg 1$, with $E=k x_{0}^{2} / 2$ and $\beta=1 / k_{B} T$ the inverse temperature. This is because the probability of multiple crossings in $\pm x_{0}$ is negligible for high barriers.

In Ref. ${ }^{18}$ the TPT distribution was calculated for a Markovian particle with inertia. It was shown that both in the inertial and overdamped cases the TPT distribution assumes the general form 18

$$
p_{T P}(t)=-\frac{2}{\sqrt{\pi}} \frac{\dot{G}(t) e^{-G^{2}(t)}}{1-\operatorname{Erf}(\sqrt{\beta E})} .
$$

where $\dot{G} \equiv d G / d t$ and

$$
G(t) \equiv \frac{x_{0}-\bar{x}(t)}{\sqrt{2 \sigma^{2}(t)}} .
$$

In the previous equation

$$
\begin{aligned}
\bar{x}(t) & \equiv\langle x(t)\rangle \\
\sigma^{2}(t) & \equiv\left\langle(x(t)-\bar{x}(t))^{2}\right\rangle
\end{aligned}
$$

are the mean and variance of the process.

In the overdamped case the function $G(t)$ assumes a simple form

$$
G(t)=\sqrt{\beta E} \sqrt{\frac{e^{\Omega t}+1}{e^{\Omega t}-1}}
$$

where $\Omega=k / \gamma$ and $\gamma$ is the friction coefficient. The $G(t)$ in the inertial case is more complex and is given in Ref. 18

For short times $G(t) \approx \sqrt{2} x_{0} / \sigma(t)$, which diverges as a consequence of the the initial condition $x(0)=-x_{0}$, implying $\sigma(t) \rightarrow 0$. This leads to a TPT distribution vanishing with an essential singularity as $t \rightarrow 0$. In the Markovian case the behavior was found to be different in the overdamped $p_{\mathrm{TP}}(t) \sim \exp \left(-x_{0}^{2} / D t\right)$ and inertial $p_{\mathrm{TP}}(t) \sim \exp \left(-2 \beta m x_{0}^{2} / t^{2}\right)$ cases ${ }^{18}$ (here $m$ is the particle mass and $D=1 /(\beta \gamma)$ the diffusion coefficient). At long times $G(t)$ converges 
to a constant in both cases, while its derivative decays exponentially $\dot{G}(t) \sim \exp (-\lambda t)$, where $\lambda^{-1}$ is the longest relaxation time of the process $(\lambda=\Omega$ in the overdamped limit (5)). This leads to an exponential decay $p_{\mathrm{TP}}(t) \sim \exp (-\lambda t)$ for the long time behavior of the distribution both in the overdamped and inertial case. ${ }^{18}$ In the next Section we compute $G(t)$ for a process with correlated and active noise and discuss the TPT distribution obtained from it.

\section{Memory effects in transition path times}

A reaction coordinate $x$ is by definition a slow variable for which standard statistical mechanical arguments show that its time evolution is given in terms of a generalized Langevin equation. $\frac{\sqrt{29}}{}$ For a parabolic barrier in the overdamped case this equation takes the form

$$
\int_{0}^{t} K(t-\tau) \dot{x}(\tau) d \tau=k x(t)+\xi(t)
$$

Here $K(t)$ is a memory kernel. The noise $\xi(t)$ is assumed to be a Gaussian process with average zero and a correlation that in equilibrium is related to $K(t)$ by the fluctuation-dissipation theorem

$$
\left\langle\xi(t) \xi\left(t^{\prime}\right)\right\rangle=k_{B} T K\left(\left|t-t^{\prime}\right|\right)
$$

We focus here on a power law memory kernel

$$
K(t)=\frac{\eta_{\alpha} t^{-\alpha}}{\Gamma(1-\alpha)}
$$

where $0<\alpha \leq 1$ and where, following Ref., 27 we define the generalized friction coefficient as $\eta_{\alpha}=\gamma \Gamma(3-\alpha)$. In the limit $\alpha \rightarrow 1^{-}$, the $\Gamma$ function in the denominator becomes singular and $K(t)=2 \gamma \delta(t)$, i.e. one recovers the Markovian (memoryless) dynamics. Power law kernels are found, for instance, in the dynamics of polymers which are characterized by a longest relaxation time $\tau_{R}$. While on time scales much larger than $\tau_{R}$ the effects of memory on the motion of a reaction coordinate can be neglected, these are strongly influencing the polymer dynamics for
$t<\tau_{R}$. Polymers have a memory kernel $K(t)$ that, for $t<\tau_{R}$, can be well approximated by a power law. 30131 This power law behavior is a characteristic of systems with a broad spectrum of relaxation times.

The generalized Langevin equation (6) with the kernel (8) is a linear equation which can be solved using Laplace transforms. The initial condition is $x(t=0)=-x_{0}$. As explained in the previous section we do not impose specific boundary conditions in $\pm x_{0}$, an approximation which is good for steep barriers $\beta E \gg 1$. The solution of (6) is (for details see Appendix )

$$
x(t)=-x_{0} \Theta_{\alpha}(t)+\frac{1}{\eta_{\alpha}} \int_{0}^{t} \xi(t-\tau) \Psi_{\alpha}(\tau) d \tau
$$

where we introduced the functions

$$
\begin{aligned}
& \Theta_{\alpha}(t) \equiv E_{\alpha, 1}\left[(\Omega t)^{\alpha}\right] \\
& \Psi_{\alpha}(t) \equiv t^{\alpha-1} E_{\alpha, \alpha}\left[(\Omega t)^{\alpha}\right]
\end{aligned}
$$

$\left(\Omega \equiv\left(k / \eta_{\alpha}\right)^{1 / \alpha}\right.$ is the characteristic rate of the process) and where

$$
E_{\alpha, \beta}(z) \equiv \sum_{n=0}^{\infty} \frac{z^{n}}{\Gamma(\alpha n+\beta)}
$$

is the Mittag-Leffler function. 32

We assume that the noise $\xi(t)$ is Gaussian, and since the Langevin equation (6) is linear, we conclude that also $x(t)$ is Gaussian. Hence Eqs. (1) and (2) apply. One has for the average

$$
\bar{x}(t) \equiv\langle x(t)\rangle=-x_{0} \Theta_{\alpha}(t)
$$

while the variance is given by

$$
\sigma^{2}(t)=\frac{k_{B} T}{k}\left(\Theta_{\alpha}^{2}(t)-1\right)
$$

(details of the calculations are in Appendix ). Plugging in (13) and (14) in (2) we get:

$$
G(t)=\sqrt{\beta E} \sqrt{\frac{\Theta_{\alpha}(t)+1}{\Theta_{\alpha}(t)-1}}
$$

This result generalizes the memoryless case (5), which is recovered in the limit $\alpha=1$ since $\Theta_{1}(x)=E_{1,1}(x)=\exp (x)$. 

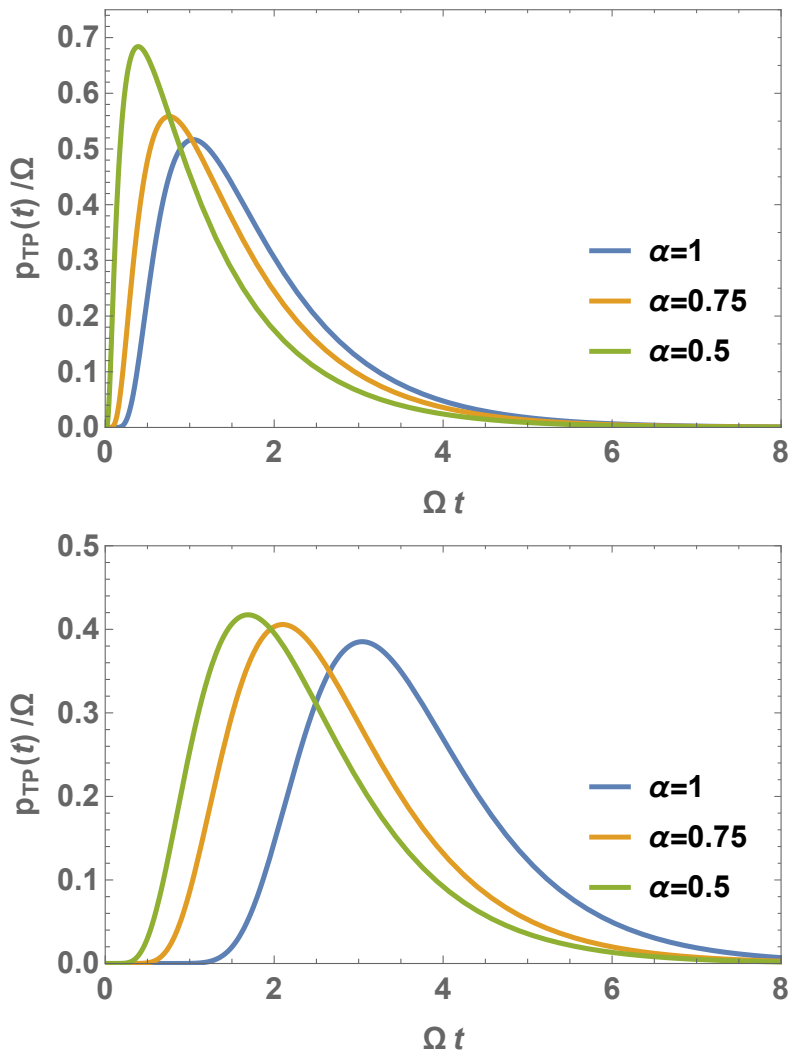

Figure 1: Transition path time distribution $p_{\mathrm{TP}}(\Omega t) / \Omega$ for $\alpha=1$ (blue), $\alpha=.75$ (orange) and $\alpha=0.5$ (green). The other parameters are $k=1, \eta_{\alpha}=10, k_{B} T=1$ and $x_{0}=\sqrt{2}$ (top), $x_{0}=\sqrt{20}$ (bottom), corresponding to $\beta E=1$ and $\beta E=10$, respectively.

Figure 1 shows plots of the transition path distribution $p_{\mathrm{TP}}(t)$ for three different values of $\alpha$ and for two different values of $k$. The transition path time (in dimensionless units) decreases with decreasing $\alpha$. We now look at the behavior of $p_{\mathrm{TP}}(t)$ for small and large times which can be obtained from the corresponding behavior of the Mittag-Leffler functions. As the Mittag-Leffler functions diverge for diverging values of their arguments (15) implies that $G(t) \rightarrow \sqrt{\beta E}$ for $t \rightarrow \infty$. For $G(t)$ one finds (for details see Appendix ).

$$
\dot{G}(t) \stackrel{t \sim \infty}{\longrightarrow}-e^{-\Omega t}
$$

which implies that $p_{\mathrm{TP}}(t)$ vanishes exponentially, as was the case for the Markovian model. $\frac{18}{18}$ This is in contrast with the conclusions of a recent paper ${ }^{20}$ where a stretched exponential decay was found. However, the

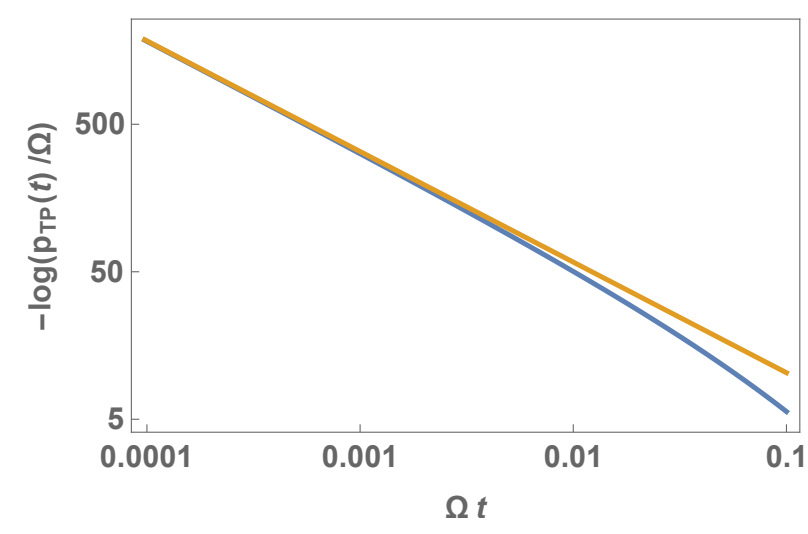

Figure 2: $\log -\log$ plot of $-\log \left(p_{\mathrm{TP}}(t) / \Omega\right)$ versus $\Omega t$ for $\alpha=0.75, k=2, x_{0}=1$ in the early time regime $\Omega t<0.1$. The blue line shows the exact expression while the orange line gives the early time approximation (17).

results of that paper where obtained from a Fokker-planck equation for systems with memory that is only correct for a linear potential, or for small times. ${ }^{33}$ Hence one cannot expect that it gives a correct asymptotic result.

For $t \rightarrow 0$, one has that $G^{2}(t) \rightarrow 2 \beta E \Gamma(1+$ $\alpha)(\Omega t)^{-\alpha}$ (see (51)) from which it follows that $\dot{G}(t) \sim t^{-\alpha / 2-1}$. The behavior of the transition path time distribution for early times is therefore determined by the essential singularity in $e^{-G^{2}(t)}$. Hence

$p_{\mathrm{TP}}(t) \stackrel{t \rightarrow 0}{\sim} e^{-G^{2}(t)}=\exp \left(-\frac{2 \beta E \Gamma(1+\alpha)}{(\Omega t)^{\alpha}}\right)$

We see that the early time behavior does depend on $\alpha$ and that the exponent could be determined from a straight line fit to a log-log plot of $-\log \left(p_{\mathrm{TP}}(t) / \Omega\right)$ versus $\Omega t$. In Fig. 2 we have made such a plot for $\alpha=0.75$ and $\beta E=1$. We find the expected power law behavior for $\Omega t<0.005$. An integration of the TPT distribution shows however that the probability that the transition path time is in this regime is extremely low $\left(\sim 10^{-43}\right)$. We therefore conclude that it is experimentally impossible to determine the exponent $\alpha$ from the early time behavior of the TPT.

We look next at the behavior of the average transition path time following the same procedure as outlined in Ref. ${ }^{18}$ As $G$ is a monotonic 
decreasing function of $t$ for the calculation it is convenient to perform a change of variable:

$$
\left\langle t_{\mathrm{TP}}\right\rangle=\int_{0}^{\infty} t p_{\mathrm{TP}}(t) d t=\frac{\int_{\sqrt{\beta E}}^{\infty} t(G) e^{-G^{2}} d G}{\int_{\sqrt{\beta E}}^{\infty} e^{-G^{2}} d G}
$$

The integral in the numerator can not be performed exactly. We can however get an approximation for $\beta E$ sufficiently large. In that limit the integrals in (18) are determined by the large $t$-limit of $G(t)$. The average TPT is then given by (for details, see Appendix )

$$
\left\langle t_{T P}\right\rangle=\frac{1}{\Omega} \log \left(2 \alpha e^{C} \beta E\right)+\mathcal{O}\left(\frac{1}{\beta E}\right)
$$

where $C \approx 0.577215$ is the Euler-Mascheroni constant (in the Markovian limit $\alpha=1$ this expression coincides with that previously obtained by Szabo ${ }^{34}$ ). In Fig. 3, we have plotted the result of a numerical calculation of the average transition path time as a function of $\beta E$ using the full expression for $G(t)$ and compared it with the approximation 19 for $\alpha=.75$. We remark that, according to $(19)$, the dimensionless average transition path time, $\Omega\left\langle t_{P T}\right\rangle$, decreases with decreasing $\alpha$ as was already evident from the plots in Fig. 1. The most likely transition path time $t_{\mathrm{TP}}^{*}$, corresponding to the maximum of a distribution is (see Appendix)

$$
t_{\mathrm{TP}}^{*}=\frac{1}{\Omega} \log (2 \alpha \beta E)+\mathcal{O}\left(\frac{1}{\beta E}\right)
$$

and show a similar logarithmic dependence on the barrier height as the average (19). The comparison between the analytical expression 20 and the numerical calculation of the maximum is shown in Fig. 3 as dashed lines.

\section{Transition path times in the presence of active forces}

The folding of a biopolymer in vivo takes place in an environment which is out of equilibrium due to the action of various ATP-dependent active processes within a cell. These processes

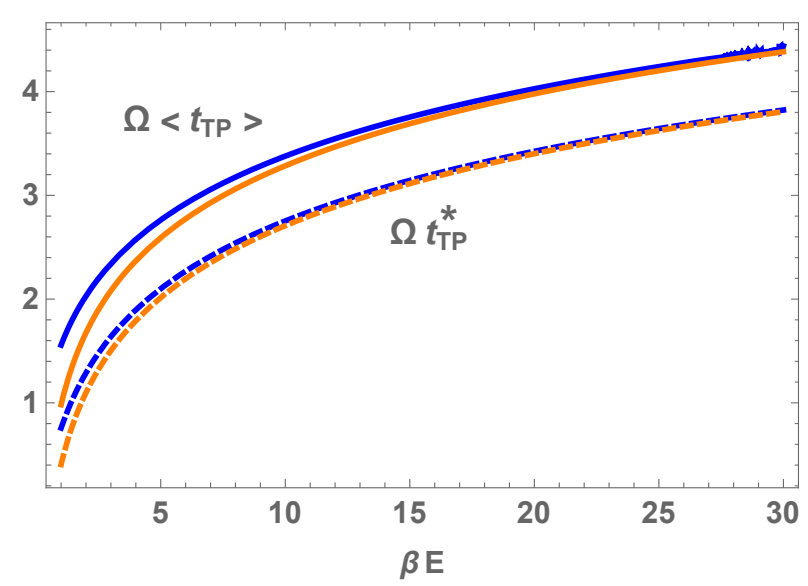

Figure 3: Average dimensionless transition path time, $\Omega\left\langle t_{T P}\right\rangle$ (solid lines), and most likely value $\Omega t_{T P}^{*}$ (dashed lines) as a function of the dimensionless energy $\beta E$ for $\alpha=0.75, k=0.1$, $\eta_{\alpha}=1$. The blue curves are obtained numerically from the exact expression for $p_{\mathrm{TP}}(t)$, while the orange curves are the approximations 190 and (20).

are known to modify the dynamics of various "probes" like microspheres ${ }^{35}$ and chromosomal loci. 3843 Typically, the active forces lead to an enhanced diffusion or even superdiffusive behavior. Similar phenomena have been observed in artificial acto-myosin networks. $\underline{44}$ It has been found that the effect of the underlying motor processes can often be described in terms of an active noise $\eta(t)$ which is correlated over the time scale $\tau_{A}$ that the motors work. These times are of the order of seconds.

In a recent study on the behavior of active Brownian particles near soft walls, the dynamics of a semiflexible polymer immersed in an environment of such particles was investigated. 49 Active Brownian particles move in a direction $\vec{e}$ which is subject to rotational Brownian diffusion. The force they produce on a (flexible) soft wall (like, for example a polymer) will therefore be exponentially correlated, where now the timescale $\tau_{A}$ of the correlation is related to the rotational diffusion constant. It was found that due to pressure instabilities, a sufficiently long polymer folds, even in the absence of interactions among the monomers.

Inspired by these two examples of folding in a non-equilibrium environment, it may be of 
interest to study also the effect of active forces on transition path times. We start from the Langevin equation

$$
\gamma \dot{x}(t)=k x(t)+\xi(t)+\eta(t)
$$

Here $\xi(t)$ is now a Markovian thermal force while $\eta(t)$ is the active noise which assume to have an exponential correlation.

$$
\left\langle\eta(t) \eta\left(t^{\prime}\right)\right\rangle=C \exp \left(-\frac{\left|t-t^{\prime}\right|}{\tau_{A}}\right)
$$

We also take $\langle\eta(t)\rangle=0$. The coefficient $C$ measures the strength of the active force. There is no associated friction force so that (21) describes a system out of equilibrium.

The solution of (21) with initial condition $x(t=0)=-x_{0}$ is

$x(t)=-x_{0} e^{\Omega t}+\frac{1}{\gamma} \int_{0}^{t} e^{\Omega\left(t-t^{\prime}\right)}\left(\xi\left(t^{\prime}\right)+\eta\left(t^{\prime}\right)\right) d t^{\prime}$

From this we find that the deterministic motion is

$$
\bar{x}(t)=\langle x(t)\rangle=-x_{0} e^{\Omega t}
$$

while the variance of the position is given by

$$
\begin{aligned}
\sigma^{2}(t) & =\left\langle(x(t)-\bar{x}(t))^{2}\right\rangle \\
& =\left(\frac{k_{B} T}{k}+\frac{C \tau_{A} \Omega}{k^{2}\left(1-\tau_{A} \Omega\right)}\right)\left(e^{2 \Omega t}-1\right) \\
& +\frac{2 C \tau_{A}^{2} \Omega^{2}}{k^{2}\left(\tau_{A}^{2} \Omega^{2}-1\right)}\left(e^{2 \Omega t}-e^{\left(1-1 / \Omega \tau_{A}\right) \Omega t}\right)
\end{aligned}
$$

It is convenient to describe the escape over the parabolic in terms of an effective temperature as was done in a study of the motion of colloids in active bath of bacteria and in the presence of a confining harmonic potential. 50

Asymptotically $\sigma^{2}(t) e^{-2 \Omega t}$ goes to a constant which can be used to define this effective temperature $T^{\star}$

$$
\begin{aligned}
\lim _{t \rightarrow \infty} \sigma^{2}(t) e^{-2 \Omega t} & =\frac{1}{k}\left[k_{B} T+\frac{C \tau_{A} \Omega}{k\left(\tau_{A} \Omega+1\right)}\right] \\
& \equiv \frac{k_{B} T^{\star}}{k}
\end{aligned}
$$

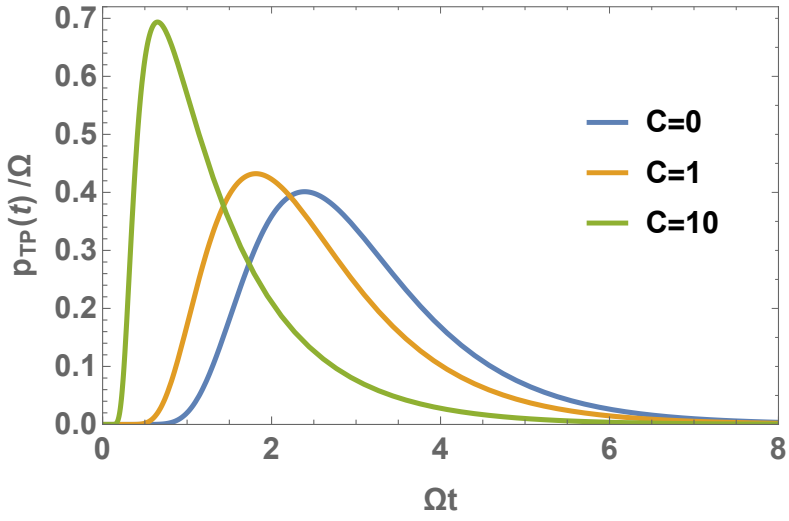

Figure 4: Transition path time distribution $p_{\text {TP }}(\Omega t) / \Omega$ for $C=0$ (blue), $C=1$ (orange) and $C=10$ (green) and for $k=0.1, \eta_{\alpha}=$ $1, x_{0}=10, k_{B} T=1, \tau_{A}=1$.

The effective temperature takes over the role of the physical temperature in the transition path time distribution. Going through the calculations of Ref. ${ }^{18}$ we find that in this case

$$
p_{\mathrm{TP}}(t)=-\frac{2}{\sqrt{\pi}} \frac{\dot{G}(t) e^{-G^{2}(t)}}{1-\operatorname{Erf}\left(\sqrt{\beta^{\star} E}\right)}
$$

where $G(t)$ is given by (2) and $\beta^{\star}=1 / k_{B} T^{\star}$.

From these results one can find that at early times, the transition path time is again governed by the essential singularity in $e^{-G^{2}(t)}$ whose form is not modified by the active forces. The late time decay is governed by $\dot{G}(t)$ which decays exponentially. The only change is in the prefactor of the exponential which now involves the effective temperature

$$
\dot{G}(t) \sim-\sqrt{\beta^{\star} E} \Omega e^{-\Omega t} \quad(t \rightarrow \infty)
$$

Finally, in the expression for the average transition path time, the effective temperature also replaces the real temperature

$$
\left\langle t_{T P}(t)\right\rangle=\Omega^{-1} \log \left(2 e^{C} \beta^{\star} E\right)
$$

Since $\beta^{\star}<\beta$, the addition of active forces leads to a decrease of the average transition path time. In Fig. 4, we have plotted some distributions where it can be seen that indeed the average transition path time decreases if the effective temperature (here tuned by changing $C$ at fixed $\tau_{A}$ ) increases. 
As can be seen from the expression of the effective temperature, the dependence on $\tau_{A}$ is weak once it becomes bigger then $\Omega^{-1}$ (the other time scale in problem). This can also be seen in Fig. 5 where $C$ is fixed and $\tau_{A}$ is increased from values below to values above $\Omega^{-1}$

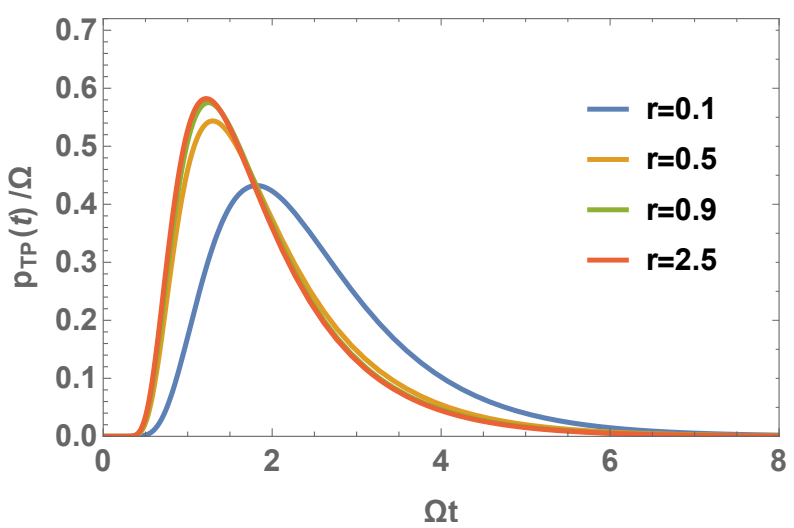

Figure 5: Transition path time distribution $p_{T P}(\Omega t) / \Omega$ for $r=0.1$ (blue), $r=0.5$ (orange) and $r=0.9$ (green) and $r=2.5$ (red) and for $k=0.1, \eta_{\alpha}=1, x_{0}=10, k_{B} T=1, C=1$. Here $r=\Omega \tau_{A}$ is the ratio of the two timescales in the problem.

\section{Discussion}

Conformational transitions of molecular systems between two different states are governed by two time scales. The Kramers time corresponds to the typical time spent in a given conformation (the dwell time), while the transition path time characterizes the actual duration of the transition. Transition path times, which have been measured in proteins and nucleic acids folding experiments during the past decade, ${ }^{3,6] 34}$ can be a few orders of magnitudes shorter than Kramers' times.

In this paper we have analyzed the TPT distribution of a one dimensional stochastic particle undergoing Langevin dynamics and crossing a parabolic barrier. We investigated the effects of memory and non-equilibrium thus extending previous analysis. $10 \mid 18$ As the barrier is parabolic, the associated Langevin equation is linear and hence exactly solvable in the case of free boundary conditions. This solution is expected to approximate very well the absorbing boundary case for steep barriers.

In Ref. 19 the effect of memory on transition path times was also investigated. The TPTdistribution was derived for an arbitrary memory kernel starting from an Hamiltonian formulation in which the particle dynamics is coupled to a bath of harmonic oscillators. 19 We expect that the expressions reported in ${ }^{19}$ will agree with our results in the case of overdamped dynamics with power-law memory in the limit of high barriers. Our result for the power law kernel has the advantage that it is simple and of the same form as in the Markovian case. We expect it to be easier to compare with experiments.

\section{Long time limit of transition path time distribution: why exponen- tial decay?}

We have found that the asymptotic decay of the transition path time distribution remains exponential for both cases investigated and therefore has a remarkable universal behavior. This contrasts with the conclusions of Ref. ${ }^{20}$ In that work, which employed a Fokker-Planck equation with a time dependent diffusion constant $D(t) \sim t^{\alpha-1}$, it was argued that the large time decay of the TPT is stretched exponential. However, it was shown that for a particle in a harmonic potential the correct expression for $D(t)$ coincides with that used in ${ }^{20}$ only for short times. 33 This suggests that the asymptotic stretched exponential behavior reported in ${ }^{20}$ cannot be trusted. A diffusion constant $D(t) \sim t^{\alpha-1}$ was also derived for a particle under constant force. $\underline{33}$

To get some more insights about the differences in the effect of memory kernels in the constant force and the parabolic barrier case let us consider the following equation

$$
\int_{0}^{t} K(t-\tau) \dot{x}(\tau) d \tau=f
$$

which describes the average motion of particle driven by a constant force $f$ in a medium char- 
acterized by the exponent $\alpha$. Using Laplace transforms we find $x(t)-x(0) \sim t^{\alpha}$, with the normal drift $x(t)-x(0) \sim t$ recovered in the Markovian limit $\alpha \rightarrow 1$. This behavior can be deduced from an effective medium description

$$
\Gamma_{\text {eff }}(t) \dot{x}(t) \sim f
$$

where the effective friction $\Gamma_{\text {eff }}(t) \sim t^{-\alpha+1}$, as expected from the time integral of memory kernel, grows with time due to memory effects (the result is consistent with the Einstein relation for the diffusion constant discussed in $\left.{ }^{33}\right)$. This indicates the velocity $\dot{x}(t) \sim f t^{\alpha-1}$ decreases with time, hence, the anomalous drift. In the parabolic barrier there is, however, a crucial difference. The effective medium description would give

$$
\Gamma_{\text {eff }}(t) \dot{x}(t) \sim k x(t)
$$

with solution

$$
x(t)=-x_{0} \exp \left[(\Omega t)^{\alpha}\right]
$$

which is a stretched exponential behavior. This is not consistent with the exact solution of the generalized Langevin equation discussed in this paper, which yields for the average position an exponentially growing function at long times (obtained from the asymptotic behavior of the Mittag-Leffler function of (13)).

To understand this apparent paradox, we point out that the effective friction argument would be valid for a process in which the velocity $\dot{x}(t)$ is a slowly varying function. For a self-similar process, where the velocity changes according to a power law $\dot{x}(t) \sim t^{-\gamma}$, the coarse grained variable $\int_{0}^{t} d t^{\prime} \dot{x}\left(t^{\prime}\right) / t \sim t^{-\gamma}$ by time average behaves similarly with the original variable. In such a case, the effective friction argument should work to get the correct scaling behavior.

However, in the parabolic barrier crossing, the velocity increases rapidly (exponentially), therefore, the contribution from the memory kernel integral is dominated by the most recent term only. This implies that in the long time scale, we should expect an effective description, in which the system feels only the instantaneous response, hence our effective equation is

$$
\tilde{\gamma} \dot{x}(t)=k x(t)
$$

where $\tilde{\gamma}$ is a renormalized friction coefficient. Our argument suggests that it is this renormalization that is behind the universal exponential decay in the long time limit of transition path time distribution. This asymptotic behavior sets already in at $\Omega t \simeq 1.5$ as seen numerically (Fig. 1).

\section{Comparison with experiments: possible implications}

Differently from the Kramers' time, which is characterised by an exponential dependence on the barrier height $E$, the average TPT in the overdamped limit scales logarithmically $\left\langle t_{\mathrm{TP}}\right\rangle \sim \log (\beta E)$, where $\beta$ is the inverse temperature. We have shown here that the logarithmic dependence also holds in the presence of memory effects or of active forces. The effect of the active forces is simply to increase the temperature to a higher effective one. Hence the average TPT will always decrease in this case. Memory also decrease the average TPT when measured in dimensionless units.

We believe that our results are helpful in interpreting experimental results. Indeed the barrier height as determined from a comparison between experiments and a model for diffusion in a parabolic potential without memory terms gave values that were much lower than those determined by other means. Our calculations have shown that both memory effects and nonequilibrium effects can have the same effect as lowering the potential barrier. While in current experiments non-equilibrium effects almost surely play no role they could certainly be of relevance inside the cellular environment. The experimental results on DNA-hairpins and proteins can however be understood from a model with memory. Indeed, in that case we predict that there are more short transit times than in a model without memory (when time is measured in rescaled unit $\Omega t$ ). This is indeed what is found experimentally. Further research will have to show whether this qualitative agree- 
ment can be made more quantitative.

Acknowledgement Discussions with $\mathrm{M}$. Caraglio and M. Laleman are gratefully acknowledged.

\section{Appendix A: Power law memory kernel}

The solution of the generalized Langevin equation (6) with power law memory kernel is obtained by performing its Laplace transform

$$
\widetilde{K}(s)\left(s \widetilde{x}(s)+x_{0}\right)=k \widetilde{x}(s)+\widetilde{\xi}(s)
$$

where $\tilde{f}(s)$ indicates the Laplace transform of the function $f(t)$. To obtain the previous equation we used the convolution theorem (the Laplace transform of a convolution product is the product of the Laplace transforms) and the fact that the Laplace transform of a derivative is

$$
\widetilde{\dot{f}}=s \tilde{f}(s)-f(0)
$$

(in our case the initial condition is $x(0)=-x_{0}$ ). Solving (33) we get

$$
\widetilde{x}(s)=\frac{-x_{0} \widetilde{K}(s)}{s \widetilde{K}(s)-k}+\frac{\widetilde{\xi}(s)}{s \widetilde{K}(s)-k}
$$

The Laplace transform of the power law kernel (8) is

$$
\widetilde{K}(s)=\gamma s^{\alpha-1}
$$

therefore Eq. (35) takes the form

$$
\widetilde{x}(s)=\frac{-x_{0} s^{\alpha}}{s\left(s^{\alpha}-k / \gamma\right)}+\frac{1}{\gamma} \frac{\widetilde{\xi}(s)}{s^{\alpha}-k / \gamma}
$$

To perform the inverse transform we use the following relation

$$
\int_{0}^{\infty} d t e^{-t s} t^{\beta-1} E_{\alpha, \beta}\left(a t^{\alpha}\right)=\frac{s^{\alpha}}{s^{\beta}\left(s^{\alpha}-a\right)}
$$

where $E_{\alpha, \beta}(z)$ is known as Mittag-Leffler function. ${ }^{32}$ To handle the two terms in the left hand side of (37) one can use (38) with $\beta=1$ and $\beta=\alpha$. For this purpose it is convenient to introduce the functions

$$
\begin{aligned}
\Theta_{\alpha}(t) & \equiv E_{\alpha, 1}\left[(\Omega t)^{\alpha}\right] \\
\Psi_{\alpha}(t) & \equiv t^{\alpha-1} E_{\alpha, \alpha}\left[(\Omega t)^{\alpha}\right]
\end{aligned}
$$

where $\Omega \equiv(k / \gamma)^{1 / \alpha}$ is a characteristic rate of the process. Inverting (37) we get

$$
x(t)=-x_{0} \Theta_{\alpha}(t)+\frac{1}{\gamma} \int_{0}^{t} \xi(t-\tau) \Psi_{\alpha}(\tau) d \tau
$$

Averaging over noise we get the average position, or equivalently the deterministic solution

$$
\bar{x}(t)=-x_{0} \Theta_{\alpha}(t)
$$

while the variance (4) is

$$
\begin{aligned}
\sigma^{2}(t) & =\frac{k_{B} T}{\gamma} \int_{0}^{t} d \tau d \sigma \frac{|\tau-\sigma|^{-\alpha}}{\Gamma(1-\alpha)} \Psi_{\alpha}(\tau) \Psi_{\alpha}(\sigma) \\
& =\frac{k_{B} T}{k}\left(\Theta_{\alpha}^{2}(t)-1\right)
\end{aligned}
$$

(the details of the calculation of this integral are given in Appendix ). We finally combine the above results to find $G(t)$, see (2)

$$
G(t)=\frac{x_{0}-\bar{x}(t)}{\sqrt{2 \sigma^{2}(t)}}=\sqrt{\beta E} \sqrt{\frac{\Theta_{\alpha}(t)+1}{\Theta_{\alpha}(t)-1}}
$$

where $E=k x_{0}^{2} / 2$ is the barrier height. This proves Eq. 15) of the main text.

The Mittag-Leffler function behaves asymptotically as 32

$$
E_{\alpha, \beta}(z) \stackrel{z \rightarrow \infty}{\longrightarrow} \frac{1}{\alpha} z^{(1-\beta) / \alpha} \exp \left(z^{1 / \alpha}\right)
$$

which implies

$$
\Theta_{\alpha}(t) \stackrel{t \rightarrow \infty}{\longrightarrow} \frac{1}{\alpha} \exp (\Omega t)
$$

Hence

$$
\begin{aligned}
& G(t) \stackrel{t \rightarrow \infty}{\longrightarrow} \sqrt{\beta E} \\
& \dot{G}(t) \stackrel{t \rightarrow \infty}{\longrightarrow}-\sqrt{\beta E} \alpha \Omega \exp (-\Omega t)
\end{aligned}
$$

For small arguments, the Mittag-Leffler func- 
tion behaves as

$$
E_{\alpha, \beta}(z)=1+\frac{z^{\alpha}}{\Gamma(\alpha+\beta)}+\cdots
$$

hence

$$
\Theta_{\alpha}(t)=1+\frac{(\Omega t)^{\alpha}}{\Gamma(1+\alpha)}+\cdots
$$

This implies that $G(t)$ diverges for small $t$

$$
G(t) \sim(\Omega t)^{-\alpha / 2}
$$

\section{The average transition path time}

To calculate the average TPT we follow the calculation outlined in: $\frac{18}{18}$

$$
\left\langle t_{T P}\right\rangle=\int_{0}^{\infty} t p_{T P}(t) d t=\frac{\int_{\sqrt{\beta E}}^{\infty} t(G) e^{-G^{2}} d G}{\int_{\sqrt{\beta E}}^{\infty} e^{-G^{2}} d G}
$$

where we have made the change of variables $G^{\prime} d t=d G$ and used the definition of the error function. The integral in the numerator can not be performed exactly. We can get an approximation for $\beta E \gg 1$ where the integrals are determined by the large $t$-limit of $G(t)$. From (39), 44) and (45) one gets for $t$ large

$$
G(t)=\sqrt{\beta E}\left[1+\alpha e^{-\Omega t}+\ldots\right]
$$

which can be inverted to

$$
t=\frac{1}{\Omega}\left(\log \alpha-\log \left(\frac{G}{\sqrt{\beta E}}-1\right)\right)
$$

If we insert (54) into (52) and make also here an expansion for large $\beta E$ we finally get

$$
\left\langle t_{T P}\right\rangle=\frac{1}{\Omega} \log \left(2 \alpha e^{C} \beta E\right)+O\left(\frac{1}{\beta E}\right)
$$

where $C \approx 0.577215$ is the Euler-Mascheroni constant.

\section{The most likely transition path time}

Another interesting quantity we can infer from the results is $t_{\mathrm{TP}}^{*}$, the most likely value of the
TPT. This is obtained by solving the equation

$$
\frac{d p_{\mathrm{TP}}}{d t}=0
$$

which from (1) implies $\ddot{G}=2 G \dot{G}^{2}$ (where the dot indicates the time derivative), or using (15):

$\dot{\Theta}_{\alpha}^{2}-\ddot{\Theta}_{\alpha}\left(\Theta_{\alpha}^{2}-1\right)+2 \Theta_{\alpha} \dot{\Theta}_{\alpha}^{2}=2 \beta E \dot{\Theta}_{\alpha}^{2} \frac{\Theta_{\alpha}+1}{\Theta_{\alpha}-1}$

Using the asymptotic $t \rightarrow+\infty$ expansion 46 one has

$$
\dot{\Theta}_{\alpha}(t) \sim \frac{\Omega}{\alpha} \exp (\Omega t), \quad \ddot{\Theta}_{\alpha}(t) \sim \frac{\Omega^{2}}{\alpha} \exp (\Omega t)
$$

and to leading order in $\beta E$ the solution of (57) becomes

$$
t_{\mathrm{TP}}^{*}=\frac{1}{\Omega} \log (2 \alpha \beta E)
$$

\section{Appendix B: Integral (43)}

To compute the integral 43 we start from the double Laplace transform of the function $\Theta_{\alpha}(|\tau-\sigma|)$. We have

$$
f\left(s, s^{\prime}\right) \equiv \int_{0}^{+\infty} \int_{0}^{+\infty} d \tau d \sigma e^{-s \tau-s^{\prime} \sigma} \Theta_{\alpha}(|\tau-\sigma|)
$$

To get rid of the absolute value we split the integral in two domains so to obtain

$$
\begin{aligned}
& f\left(s, s^{\prime}\right)=\int_{0}^{+\infty} d \tau \int_{\tau}^{+\infty} d \sigma e^{-s \tau-s^{\prime} \sigma} \Theta_{\alpha}(\sigma-\tau) \\
& +\int_{0}^{+\infty} d \sigma \int_{\tau}^{+\infty} d \tau e^{-s \tau-s^{\prime} \sigma} \Theta_{\alpha}(\tau-\sigma) \\
& =\frac{1}{s+s^{\prime}}\left[\frac{1}{s^{\prime}\left(1-\left(\Omega / s^{\prime}\right)^{\alpha}\right)}+\frac{1}{s\left(1-(\Omega / s)^{\alpha}\right)}\right]
\end{aligned}
$$

The integrals can be easily computed using a change of variables and the property (38). The 
above expression can be rearranged as follows

$$
\begin{aligned}
& f\left(s, s^{\prime}\right)=\frac{1}{s s^{\prime}\left(1-\left(\Omega / s^{\prime}\right)^{\alpha}\right)\left(1-(\Omega / s)^{\alpha}\right)}- \\
& \frac{\Omega^{\alpha}}{\left(s s^{\prime}\right)^{\alpha}\left(1-\left(\Omega / s^{\prime}\right)^{\alpha}\right)\left(1-(\Omega / s)^{\alpha}\right)} \frac{s^{\alpha-1}+s^{\prime \alpha-1}}{s+s^{\prime}}
\end{aligned}
$$

The double inverse Laplace transform of the first term is easy as this term is the product of a function of $s$ and a function of $s^{\prime}$. One has two independent inverse Laplace transform and from (38) one sees that this generates $\Theta_{\alpha}(\tau) \Theta_{\alpha}(\sigma)$.

The second term in 62 is a product of two fractions. In the first one, one recognises the double Laplace transform of $\Omega^{\alpha} \Psi_{\alpha}(\tau) \Psi_{\alpha}(\sigma)$. For the second one we use

$\int_{0}^{+\infty} d \tau d \sigma \frac{e^{-\sigma s-\tau s^{\prime}}|\tau-\sigma|^{-\alpha}}{\Gamma(1-\alpha)}=\frac{s^{\alpha-1}+s^{\prime \alpha-1}}{s+s^{\prime}}$

Invoking the convolution theorem of double Laplace transforms, the second term of 62 is therefore the double Laplace transform of the convolution

$\left.\frac{\Omega^{\alpha}}{\Gamma(1-\alpha)} \int_{0}^{t} \int_{0}^{t^{\prime}} d \tau d \sigma \mid \tau-\sigma\right]^{-\alpha} \Psi_{\alpha}(\tau) \Psi_{\alpha}(\sigma)$

Putting everything together we have

$$
\begin{array}{r}
\left.\frac{\Omega^{\alpha}}{\Gamma(1-\alpha)} \int_{0}^{t} \int_{0}^{t^{\prime}} d \tau d \sigma \mid \tau-\sigma\right]^{-\alpha} \Psi_{\alpha}(\tau) \Psi_{\alpha}(\sigma) \\
=\Theta_{\alpha}(t) \Theta_{\alpha}\left(t^{\prime}\right)-\Theta_{\alpha}\left(\left|t-t^{\prime}\right|\right)
\end{array}
$$

from which (43) follows by putting $t=t^{\prime}$.

\section{References}

(1) Hänggi, P.; Talkner, P.; Borkovec, M. Rev. Mod. Phys. 1990, 62, 251.

(2) Hummer, G. J. Chem. Phys. 2004, 120, 516-523.

(3) Chung, H. S.; Louis, J. M.; Eaton, W. A.
Proc. Natl. Acad. Sci. USA 2009, 106, 11837-11844.

(4) Neupane, K.; Ritchie, D. B.; Yu, H.; Foster, D. A. N.; Wang, F.; Woodside, M. T. Phys. Rev. Lett. 2012, 109, 068102.

(5) Truex, K.; Chung, H. S.; Louis, J. M.; Eaton, W. A. Phys. Rev. Lett. 2015, 115, 018101.

(6) Neupane, K.; Wang, F.; Woodside, M. T. Proc. Natl. Acad. Sci. USA 2017, 201611602.

(7) Neupane, K.; Foster, D. A.; Dee, D. R.; Yu, H.; Wang, F.; Woodside, M. T. Science 2016, 352, 239-242.

(8) Berezhkovskii, A.; Szabo, A. J. Chem. Phys. 2005, 122, 014503.

(9) Dudko, O. K.; Hummer, G.; Szabo, A. Phys. Rev. Lett. 2006, 96, 108101.

(10) Zhang, B. W.; Jasnow, D.; Zuckerman, D. M. J. Chem. Phys. 2007, 126, 074504 .

(11) Sega, M.; Faccioli, P.; Pederiva, F.; Garberoglio, G.; Orland, H. Phys. Rev. Lett. 2007, 99, 118102.

(12) Chaudhury, S.; Makarov, D. E. J. Chem. Phys. 2010, 133, 034118.

(13) Orland, H. J. Chem. Phys. 2011, 134, 174114.

(14) Kim, W. K.; Netz, R. R. J. Chem. Phys. 2015, 143, 224108.

(15) Makarov, D. E. J. Chem. Phys. 2015, 143, 194103.

(16) Daldrop, J. O.; Kim, W. K.; Netz, R. R. EPL (Europhysics Letters) 2016, 113, 18004.

(17) Berezhkovskii, A. M.; Dagdug, L.; Bezrukov, S. M. J. Phys. Chem. B 2017, 121, 5455 . 
(18) Laleman, M.; Carlon, E.; Orland, H. J. Chem. Phys 2017, 147, 214103.

(19) Pollak, E. Phys. Chem. Chem. Phys. 2016, 18, 28872.

(20) Satija, R.; Das, A.; Makarov, D. E. J. Chem. Phys. 2017, 147, 152707.

(21) Sakaue, T. Phys. Rev. E 2007, 76, 021803.

(22) Panja, D.; Barkema, G. T.; Ball, R. C. Journal of Physics: Condensed Matter 2007, 19, 432202 .

(23) Dubbeldam, J. L.; Rostiashvili, V.; Milchev, A.; Vilgis, T. A. Phys. Rev. E 2011, 83, 011802.

(24) Walter, J.-C.; Ferrantini, A.; Carlon, E.; Vanderzande, C. Phys. Rev. E 2012, 85, 031120 .

(25) Frederickx, R.; In't Veld, T.; Carlon, E. Phys. Rev. Lett. 2014, 112, 198102.

(26) Sakaue, T.; Walter, J.-C.; Carlon, E.; Vanderzande, C. Soft Matter 2017, 13, 31743181 .

(27) Vandebroek, H.; Vanderzande, C. Soft Matter 2017, 13, 2181-2191.

(28) Bechinger, C.; Di Leonardo, R.; Löwen, H.; Reichhardt, C.; Volpe, G.; Volpe, G. Rev. Mod. Phys. 2016, 88, 045006 .

(29) Zwanzig, R. Nonequilibrium Statistical Mechanics; Oxford University Press, 2001.

(30) Panja, D. J. Stat. Mech.: Theory and Experiment 2010, 2010, L02001.

(31) Saito, T.; Sakaue, T. Phys. Rev. E 2015, 92, 012601.

(32) Haubold, H. J.; Mathai, A. M.; Saxena, R. K. Journal of Applied Mathematics 2011, 2011.
(33) Goychuk, I. Adv. Chem. Phys. 2012, 150, 187.

(34) Chung, H. S.; McHale, K.; Louis, J. M.; Eaton, W. A. Science 2012, 335, 981.

(35) Caspi, A.; Granek, R.; Elbaum, M. Phys. Rev. Lett. 2000, 85, 5655.

(36) Gal, N.; Weihs, D. Cell Biochem. Biophys. 2012, 63, 199.

(37) Goldstein, D.; Elhanan, T.; Aronovitch, M.; Weihs, D. Soft Matter 2013, 9, 7167.

(38) Weber, S.; Spakowitz, A.; Theriot, J. Proc. Natl. Acad. Sci. USA 2012, 109, 7338.

(39) Javer, A.; Long, Z.; Nugent, E.; Grisi, M.; Siriwatwetchakul, K.; Dorfman, K.; Cicuta, P.; Cosentino Lagomarsino, M. Nat. Commun. 2013, 4, 3003.

(40) Javer, A.; Kuwada, N.; Long, Z.; Benza, V.; Dorfman, K.; Wiggins, P.; Cicuta, P.; Cosentino Lagomarsino, M. Nat. Commun. 2014, 5, 3854.

(41) Zidovska, A.; Weitz, D.; Mitchinson, T. Proc. Natl. Acad. Sci. USA 2013, 110, 15555.

(42) Sakaue, T.; Saito, T. Soft Matter 2017, $13,81$.

(43) Arai, R.; Sugawara, T.; Sato, Y.; Minakuchi, Y.; Toyoda, A.; Nabeshima, K.; Kimura, H.; Kimura, A. Scientific Reports 2017, $7,3631$.

(44) Soares e Silva, M.; Stuhrmann, B.; Betz, T.; Koenderink, G. New J. Phys. 2014, 16, 075010.

(45) Stuhrmann, B.; Soares e Silva, M.; Depken, M.; MacKintosh, F.; Koenderink, G. Phys. Rev. E 2012, 86, 020901(Rà.

(46) Toyota, T.; Head, D.; Schmidt, C.; Mizuno, D. Soft Matter 2011, 7, 3243. 
(47) Brangwynne, C.; Koenderink, G.; MacKintosh, F.; Weitz, D. Phys. Rev. Lett. 2008, 100, 118104.

(48) Fakhri, N.; Wessel, A.; Willms, C.; Pasquali, M.; Klopfensstein, D.; MacKintosh, F.; Schmidt, C. Science 2014, 344, 1031.

(49) Nikola, N.; Solon, A. P.; Kafri, Y.; Kardar, M.; Tailleur, J.; Voituriez, R. Phys. Rev. Lett. 2016, 117, 098001.

(50) Maggi, C.; Paoluzzi, M.; Pellicciotta, N.; Lepore, A.; Angelani, L.; Di Leonardo, R. Phys. Rev. Lett. 2014, 113, 238303. 